TITLE:

\title{
Shapes of laser-produced CsH particles
}

\section{$\operatorname{AUTHOR}(\mathrm{S})$ :}

Tanaka, T.; Mitsui, T.; Sugiyama, K.; Kitano, M.; Yabuzaki, T.

\section{CITATION:}

Tanaka, T. ... [et al]. Shapes of laser-produced CsH particles. Physical Review Letters 1989, 63(13): 1390-1392

ISSUE DATE:

1989-09

URL:

http://hdl.handle.net/2433/152442

RIGHT:

c 1989 The American Physical Society 


\title{
Shapes of Laser-Produced CsH Particles
}

\author{
T. Tanaka, ${ }^{(1)}$ T. Mitsui, ${ }^{(1)}$ K. Sugiyama, ${ }^{(2)}$ M. Kitano, ${ }^{(3)}$ and T. Yabuzaki ${ }^{(1)}$ \\ (1) Department of Physics, Kyoto University, Kyoto 606, Japan \\ (2) National Research Laboratory of Meterology, Tsukuba, Ibaraki 305, Japan \\ (3) Department of Electronics, Kyoto University, Kyoto 606, Japan
}

(Received 8 June 1989)

\begin{abstract}
Shapes of laser-produced alkali-hydride crystalline particles (laser snow) are observed for the first time. Particles are suspended by an electric field so as to grow large enough to be observed by an optical microscope. It is found that the particles are single crystals and have particular shapes depending on the gas temperature and the laser-light intensity.
\end{abstract}

PACS numbers: $68.70 .+\mathrm{w}$

In 1975, Tam Moe, and Happer ${ }^{1}$ found the interesting phenomenon that micron-size particles are produced within a laser beam when Cs atoms mixed with hydrogen and rare gases are excited by the laser light to states such as the $7 P$ or $9 D$. The particles produced are called "laser snow," because they fall down by gravity; they can be observed with the naked eye, and they are expected to be crystals because of the formation of white powder on the bottom of the cell. These particles are known to be produced by the condensation of $\mathrm{CsH}$ molecules, which are produced by the chemical reaction between the excited-state $\mathrm{Cs}$ atom, $\mathrm{Cs}^{*}$, and ground-state $\mathrm{H}_{2}$. Two possible routes for production of $\mathrm{CsH}$ molecules have been considered; a direct ${ }^{1,2}$ reaction where $C s^{*}$ reacts directly with ground-state $\mathrm{H}_{2}$, and indirect ${ }^{3}$ processes where $\mathrm{Cs}^{*}$ collisionally excites $\mathrm{H}_{2}$ to some vibrational state in the ground electronic state which then reacts with the ground-state Cs. In the present stage we do not know which is predominant, but in any case the production rate is very high, so that the number density of $\mathrm{CsH}$ easily exceeds the critical value of condensation.

In our previous experiments, it was found that such particles were produced by the laser excitation of all the alkali-metal atoms tested, and even of alkali-metal dimers. ${ }^{4}$ Thermodynamical consideration ${ }^{5}$ shows that when the particles are produced at a relatively high temperature $\left(\geq 280^{\circ} \mathrm{C}\right.$ ), they cool the environmental gas by absorption of latent heat through the evaporation of hydrogen from the surface. In this way, the resemblance of the nature of the laser snow to that of natural snow has deepened.

In this paper we report on the first observation of the shapes of alkali-hydride laser snow. Since laser snow is made up of growing crystals, they are expected to have particular shapes which are different from the equilibrium form known to be cubic and dependent on the temperature and supersaturation of molecules, from the analogy with natural snow. However, no one has observed the shapes so far because of the small size of the particles. The size of the particles can easily be measured from their falling velocity in a buffer gas, similarly to Millikan's oil-drop experiment, and the maximum size observed so far is approximately $1 \mu \mathrm{m}$, which is about the resolution of an ordinary optical microscope. Recently, Kim and Fedele ${ }^{6}$ pointed out the breakdown of Millikan's law, but in the present case, the deviation from it is not expected to be significant because the diffusion length is much larger than the falling velocity. When the particles grow larger, they fall from the laser beam even in the presence of a relatively high-pressure buffer gas, so that, in order to get particles large enough to be observed through an optical microscope, it is necessary to suspend them within the laser beam against the force of gravity by some means. Fortunately the particles have a large amount of positive charge, typically from $10^{3}$ to $10^{4}$ times as large as an elementary charge. The charge is known to be produced by the emission of photoelectrons by the excitation laser light with wavelengths shorter than $500 \mathrm{~nm}$. Alkali-hydride crystals are well-known materials with a high photoelectron emissivity for light in the green-violet region. ${ }^{7}$ In the present work, we suspended the $\mathrm{CsH}$ laser snow in a laser beam by applying a vertical electric field.

The experimental setup is very simple as shown schematically in Fig. 1. The cell used here is cylindrical with a $5-\mathrm{cm}$ length and a $3-\mathrm{cm}$ diameter, and is made of alkali-resistant glass (Corning 1720). The cell has a pair of electrodes made of tungsten. In Fig. 1 linear electrodes are shown. We have also used ring-shaped ones in order to avoid the diffusion of the suspended particles in

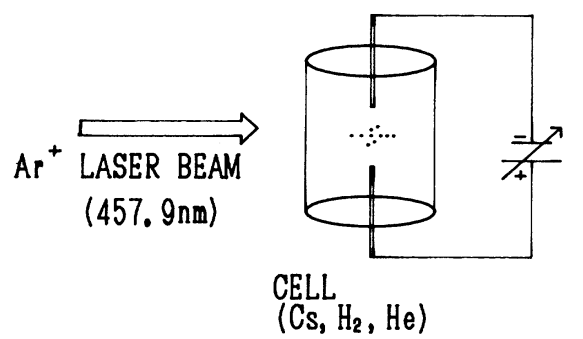

FIG. 1. Schematic illustration of experimental setup. The 457.9-nm line from a $\mathrm{cw} \mathrm{Ar}^{+}$laser is used to excite $\mathrm{Cs}$ atoms to $7 P$ states. The cell is placed in a transparent glass oven. 
TABLE I. Size and typical shapes of suspended CsH laser snow produced at various temperatures up to $300^{\circ} \mathrm{C}$ and at laser powers of 80 and $300 \mathrm{~mW}$. At temperatures lower than $200^{\circ} \mathrm{C}$, the shapes could not be observed because of the small size $(<3 \mu \mathrm{m})$.

\begin{tabular}{|c|c|c|c|}
\hline $\begin{array}{l}\text { Temperature } \\
\left({ }^{\circ} \mathrm{C}\right)\end{array}$ & $\begin{array}{l}\text { Laser power } \\
\quad(\mathrm{mW})\end{array}$ & $\begin{array}{l}\text { Size (radius) } \\
(\mu \mathrm{m})\end{array}$ & Shapes \\
\hline 230 & 300 & 18 & Bullet shape (or pyramid) \\
\hline 250 & 80 & 21 & $\begin{array}{l}\text { Polyhedra (mostly with } \\
\text { hexagonal cross section) }\end{array}$ \\
\hline 250 & 300 & 13 & Tetrahedron, plate shape \\
\hline 300 & 80 & 400 (length) & Whisker \\
\hline
\end{tabular}

horizontal directions. Before sealing, the cell was baked at a temperature of about $450{ }^{\circ} \mathrm{C}$ for half a day and filled with a small amount of Cs metal, hydrogen gas at about 20 Torr, and helium gas at about 500 Torr as a buffer gas. The cell was placed in a transparent glass over, which was carefully designed to avoid convection in the cell. The cell temperature was controlled over the range up to $300^{\circ} \mathrm{C}$, the corresponding cesium density being up to $6 \times 10^{16} \mathrm{~cm}^{-3}$. As a light source, we used a cw $\mathrm{Ar}^{+}$laser operating selectively at $457.9 \mathrm{~nm}$, which can excite cesium atoms from the ground state $6 S_{1 / 2}$ to the second excited states $7 P_{1 / 2,3 / 2}$ through far wings of the pressure-broadened absorption lines centered at 455.5 and $459.3 \mathrm{~nm}$. The laser beam was collimated and the beam diameter at the cell was about $1 \mathrm{~mm}$. The laser power was varied up to $300 \mathrm{~mW}$.

The experimental procedure was as follows. Within a second from the irradiation of the laser beam, we could observe strong scattering of the beam by the produced particles of small size. ${ }^{8}$ Soon after, individual particles grew large enough to be identified with the naked eye, and then they begin to fall down by gravity. From the falling velocity, we estimated the particle size to be a little bit smaller than $1 \mu \mathrm{m}$. Then we applied the electric field, and adjusted the field intensity so that the particles were always suspended within the laser beam. From the field intensity for suspension, we estimated the charge to be $10^{3}-10^{4}$ in the early stage. These values agree roughly with the previous experiments. ${ }^{1,8}$ Looking at a particular group of particles, we continued to adjust carefully the electric field for 1 or $2 \mathrm{~h}$. Then, we turned off the electric field and allowed the large particles to drop from the laser beam onto the bottom of the cell. Then we took the cell out of the oven and observed the particles through the bottom window of the cell by an optical microscope.

The particles obtained in this way were larger than 10 $\mu \mathrm{m}$, particularly when they were produced at a temperature higher than about $230^{\circ} \mathrm{C}$. It was found that these particles were single crystals with characteristic shapes, different from the equilibrium form known to be cubic. Although we have not studied them systematically so far, it is apparent that the shapes, as well as the size, of the particles are strongly dependent on the cell temperature and also on the laser intensity. Table I shows rough dependences on these quantities. The characteristic shapes observed are bullet shape (or pyramid with a nearly round cross section), tetrahedron, more complicated polyhedra, flat and thin plate, and very long whisker. Figures 2(a) and 2(b) show the crystals with the shapes of the bullet (or pyramid) and polyhedron with hexagonal cross section, the sizes of which are about $15-20 \mu \mathrm{m}$ in mean radius. Figure 2 (c) shows the longest whisker crystal obtained at about $300^{\circ} \mathrm{C}$, which has a length of about $400 \mu \mathrm{m}$ and a width of about $5 \mu \mathrm{m}$.

It must be noted that these large particles were evidently produced within the laser beam and not on the surfaces of the cell nor on the electrodes. We could ascertain this from the same experiments as above but with no electric field. Although we repeated the experiment several times, no particles larger than $2 \mu \mathrm{m}$ could

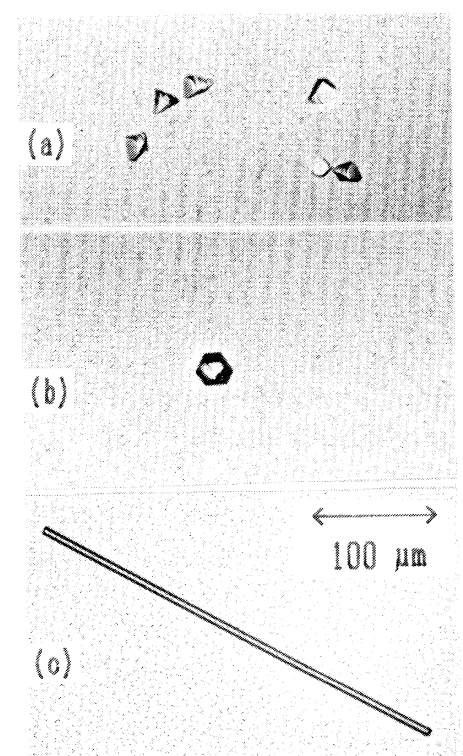

FIG. 2. Photographs of three characteristic shapes of $\mathrm{CsH}$ laser snow suspended by the electric field for about $2 \mathrm{~h}$; (a) bullet (or pyramid) shaped crystals, (b) a polyhedron with hexagonal cross section, and (c) a whisker crystal. 
be observed in this case. The small particles formed white powder on the bottom of the cell, just below the laser beam.

The growth mechanism of laser-snow particles with different shapes is not the main subject of the present paper. However, it should be noted that the particle growth is apparently due to the condensation of $\mathrm{CsH}$ molecules and is not the coalescence of particles, particularly at temperatures lower than $280^{\circ} \mathrm{C}$ in the present case. This is because of the fact that the particles have positive charges, as mentioned already, and the repulsive Coulomb forces among them are much stronger than the thermal energies. With respect to the condensation of $\mathrm{CsH}$, we found recently a "harpooning" effect of photoelectrons, which enhances considerably the growth rate of particles. Namely, the photoelectrons emitted from the particles attach to $\mathrm{CsH}$ molecules through threebody collisions and produce negative ions. The electron affinity of $\mathrm{CsH}$ molecules is known to be $0.438 \mathrm{eV} .{ }^{9}$ The negative ions are attracted to particles through a longrange Coulomb force. The harpooning effect of photoelectrons can be considered to be one possible cause of symmetry breaking in the growth of particles, because the charge distribution within particles is not expected to be uniform. (The details about the harpooning effect are to be reported elsewhere.) Our thermodynamical analysis shows that hydrogen atoms (or molecules) are weakly dissociated from the surface at a temperature of about $300^{\circ} \mathrm{C}$. Then, the top of a long crystal where surface tension is high is expected to be wet and have a high sticking probability at this temperature. So, we believe that the growth of the long crystals observed in this experiment [see Fig. 2(c)] is due to a mechanism like the vapor-liquid-solid (VLS) model, ${ }^{10}$ which was introduced to explain the growth of silicon whisker crystals in the gas phase.

The change of the shapes (habits) of natural snow from water vapor has been studied for many years, and the dependences on the temperature and supersaturation of water vapor are roughly expressed by the so-called Kobayashi diagram. ${ }^{11}$ An interesting point is that shapes similar to the ones observed in the present experiment can be seen in natural snow. For example, long needle-shaped crystals are produced at a relatively high temperature and high supersaturation, and were actually observed on the South Antarctic continent. ${ }^{12}$ Particles with a more complicated shape, such as the one corresponding to the snow flake, which we wanted to observe, are expected to be produced at a low temperature and high supersaturation, from the analogy with the change of habits of natural snow. However, at low temperature the Cs density is low (for example, Cs density at $200^{\circ} \mathrm{C}$ is $3 \times 10^{16}$ which is 1 order of magnitude lower than that at $300^{\circ} \mathrm{C}$ ), so that it is very difficult to get very high supersaturation of $\mathrm{CsH}$ molecules.

Compared with other systems that produce crystalline particles in the gas phase, the system for laser snow is very simple. In addition, the controlling parameters such as gas temperature and supersaturation (which is mainly determined by the laser intensity) can be precisely regulated. Furthermore, as mentioned already, the growth of laser-snow particles is only by the condensation of molecules and not by the coalescence of particles because of the strong Coulomb repulsive force; this may be the main reason why we could obtain single crystals only. Consequently, it can be said that the laser-snow system is a very suitable one for fundamental studies of condensation and crystallization in the gas phase. Systematic studies on the change of laser-snow shape under various conditions are now under way in our laboratory, with special interest in whether the shape changes as the particle grow or not. We are now studying quantitatively a new type of particle growth, namely, the harpooning effect of photoelectrons mentioned above.

We would like to thank W. Happer and A. C. Tam for their useful discussions and comments.

${ }^{1}$ A. Tam, G. Moe, and W. Happer, Phys. Rev. Lett. 35, 1630 (1975).

${ }^{2}$ A. C. Tam, W. Happer, and D. Siano, Chem. Phys. Lett. 49, 320 (1976).

${ }^{3}$ J. P. Visticot, M. Ferrey, J. Lozingot, and B. Sayer, J. Chem. Phys. 79, 2839 (1983).

${ }^{4}$ T. Yabuzaki, T. Sato, and T. Ogawa, J. Chem. Phys. 73, 2780 (1980).

${ }^{5}$ T. Yabuzaki, S. Curry, J. Camparo, and W. Happer, Columbia University, Department of Physics, Report No. 18, 1978 (unpublished).

${ }^{6}$ Y. W. Kim and P. D. Fedele, Phys. Rev. Lett. 48, 403 (1982).

${ }^{7}$ E. F. Seiler, Astrophys. J. 52, 129 (1920).

${ }^{8}$ T. Sato, T. Yabuzaki, and T. Ogawa, Jpn. J. Appl. Phys. 21, 1599 (1982).

${ }^{9}$ W. J. Stevens, A. M. Karo, and J. R. Hiskes, J. Chem. Phys. 74, 3989 (1981).

${ }^{10}$ R. S. Wagner and C. Ellis, Trans. Metall. Soc. AIME 233, 1053 (1965).

${ }^{11}$ T. Kobayashi, Philos. Mag. 6, 1363 (1961).

${ }^{12}$ H. Shimizu, J. Met. Soc. 41, 305 (1963). 


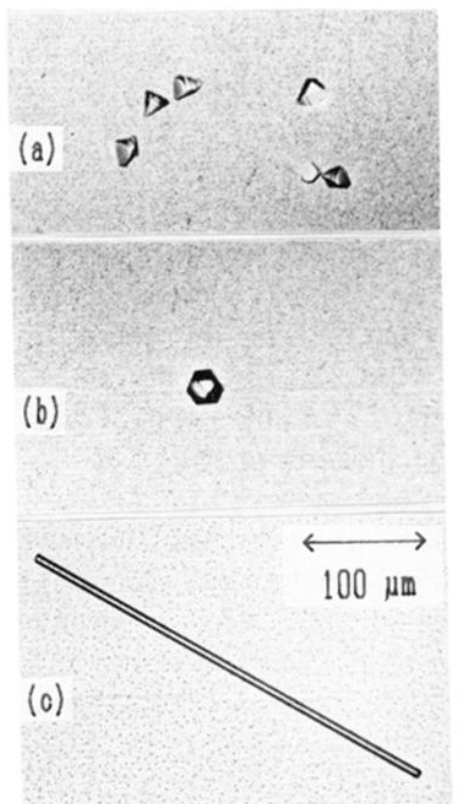

FIG. 2. Photographs of three characteristic shapes of $\mathrm{CsH}$ laser snow suspended by the electric field for about $2 \mathrm{~h}$; (a) bullet (or pyramid) shaped crystals, (b) a polyhedron with hexagonal cross section, and (c) a whisker crystal. 